\title{
Credit Risk Models with Incomplete Information*
}

\author{
Xin Guo ${ }^{\dagger} \quad$ Robert A. Jarrow ${ }^{\ddagger}$ Yan Zeng§ \\ (First Version "Information Reduction in Credit Risk Models", March 9, 2005)
}

June 18, 2008

\begin{abstract}
Incomplete information is at the heart of information-based credit risk models. In this paper, we rigorously define incomplete information with the notion of "delayed filtrations". We characterize two distinct types of delayed information, continuous and discrete: the first generated by a time change of filtrations and the second by finitely many marked point processes. This notion unifies the noisy information in Duffie and Lando (2001) and the partial information in Collin-Dufresne et al. (2004), under which structural models are translated into reduced-form intensity-based models. We illustrate through a simple example the importance of this notion of delayed information, as well as the potential pitfall for abusing the Laplacian approximation techniques for calculating the intensity process in an information-based model.
\end{abstract}

\footnotetext{
${ }^{*}$ The authors thank the Associate Editor and the two anonymous referees for their careful reading, constructive suggestions, and enlightening remarks.

${ }^{\dagger}$ Dept. of IEOR, 4173 Etcheverry Hall, UC at Berkeley. Email: xinguo@ieor.berkeley.edu. Tel: 510-642-3615. Support from NSF DMUI-0522342 is gratefully acknowledged.

$\ddagger_{451}$ Sage Hall, Johnson School of Management. Cornell University, Ithaca, NY 14853, and Kamakura Corporation.:w Email: raj15@cornell.edu. Tel: 607-255-4729.

${ }^{\S}$ Bloomberg. Email: yzeng5@bloomberg.net. Tel: 212-617-3975.
} 


\section{Introduction}

Given the size and recent growth of the credit risk markets (see Creditflux, 2004), it is not surprising that the credit risk literature has experienced a similar expansion, as evidenced by the number of books and articles published on this topic. Two types of credit risk models have been studied in the literature: structural and reduced-form. Structural models view a firm's liabilities as complex put options on the firm's assets. Therefore, modeled in this approach are the firm's liability structure and the firm's asset value process. This methodology originated with Black and Scholes (1973) and Merton (1974). In these models, the default time is usually characterized as the first hitting time of the firm's asset value to a given boundary determined by the firm's liabilities. As such, if the firm's asset value process follows a diffusion, then the default time is usually a predictable stopping time. The difficulties with the structural approach are twofold: first, the firm's asset value process is not directly observable, making empirical implementation difficult; and second, a predictable default time implies credit spreads should be near zero on short maturity debt. This second implication is well known to be inconsistent with historical market credit spread data.

In contrast, the reduced-form approach was developed precisely to avoid modeling the firm's unobservable asset value process. This approach was originated by Jarrow and Turnbull (1992, 1995), Artzner and Delbaen (1995), and Duffie and Singleton (1999). Typically, reduced-form models characterize default as the first jump time of a point process, often a Cox process (i.e., a doubly stochastic Poisson process). As such, the default time is usually a totally inaccessible stopping time, implying non-zero credit spreads for short maturity debt. A review of the credit risk literature can be found in many good books, including Ammann (2001), Bielecki and Rutkowski (2002), Duffie and Singleton (2003), Schönbucher (2003), and Lando (2004). A systematic study of the mathematical techniques used in reduced-form models is available in Elliott, Jeanblanc, and Yor (2000) and Jeanblanc and Rutkowski (2002).

As implied by the above description, structural and reduced-form models are viewed as competing paradigms. However, recent work by Duffie and Lando (2001), Collin-Dufresne, Goldstein, and Helwege (2003), Çetin et al. (2004), and Jarrow and Protter (2004) point out an intrinsic connection between these two approaches. Reduced-form models can be viewed as structural models analyzed under different information filtrations: Structural models are based on the information set available to the firm's management, which includes continuous-time observations of both the firm's asset value and liabilities; reduced-form models are based on the information set available to the market, typically including only partial observations of both the firm's asset value and liabilities. As shown in examples by the above authors, it is possible to transform a structural model with a predictable default time into a reduced-form model, with a totally inaccessible default time, by introducing "incomplete information". For instance, Duffie and Lando (2001) used a noisy and discretely observed firm asset value in a continuous-time model, while Collin-Dufresne, Goldstein, and Helwege (2003) used a simple form of delayed information in a Brownian motion model.

However, despite this recent development on information-based models, the notion of "incomplete information" has yet to be properly defined. Furthermore, to be useful for understanding and extending the existing literature, such a definition should unify the previously mentioned notions of "noisy" and "delayed" information into the same framework. Our paper is the first attempt to do this.

First, we rigorously define incomplete information via the notion of a "delayed filtration", for both discrete and continuous types. The latter is developed from the work of Jacod and Skorohod (1994) on jumping filtration of a marked point process, while the former is defined through a 
time change of the relevant filtrations. We then study the distinction between these two types of incomplete information, and illustrate in Example 2 and Example 6 how the "noisy" information in Duffie and Lando (2001) and the "delayed" information in Collin-Dufresne et al. (2004) are examples of our definitions (Section 2).

Next, we generalize under this mathematical framework both Duffie and Lando (2001) and Collin-Dufresne et al. (2003) to characterize the existence of an intensity process for any Markov model, with or without jumps. The existence of intensity process under our formulation of delayed filtration may be viewed as dynamic versions of the work in Dellacherie (1972, Chapter V, T56). Finally, we study through a simple example the importance of this notion of delayed information, as well as the potential pitfall for abusing Meyer's Laplacian approximation techniques for calculating the intensity process in an information-based models (Section 3).

Applying our theory to various representative models for the asset value process, we derive explicit formulas for the intensity process. As in earlier work, we show that incomplete information transforms a predictable default time into a totally inaccessible stopping time. In addition, we provide a characterization of the intensity process that is useful for empirical estimation (see Chava and Jarrow (2004) and Duffie and Wang (2003) for existing empirical studies). Finally, we study the relations of risky debt pricing under different filtrations: complete and incomplete (Section 4).

All proofs are given in the Appendix.

\section{Incomplete Information and Delayed Filtration: Mathematical Formulation}

With regard to incomplete information, the following two well-known cases are instrumental: the noisy and discrete accounting information in Duffie and Lando (2001) and the delayed information from continuous observations in Collin-Dufresne et al. (2003). We shall incorporate both the continuous and discrete nature of these information into our notion of "delayed filtration". Intuitively, a continuously delayed filtration allows information to flow in continuously, albeit following a time clock slower than the ordinary one. A discretely delayed filtration, on the other hand, does not allow new information to flow in between two consecutive observation times. Clearly, these two types of filtrations are distinct, as we shall formalize this difference later in this section.

\subsection{Definition of Delayed Filtration}

First, we define

Definition 1 (Continuously delayed filtration). Given a filtration $\mathbb{H}=\left(\mathcal{H}_{t}\right)_{t \geq 0}$ that is right continuous and contains all the negligible sets ${ }^{1}$. Suppose that $\left(\alpha_{t}\right)_{t \geq 0}$ is a time change of $\mathbb{H}^{2}$ so that for a.s. $\omega, \alpha_{t}(\omega) \leq t$ for all $t$. The time-changed filtration $\mathbb{F}=\left(\mathcal{F}_{t}\right)_{t \geq 0}=\left(\mathcal{H}_{\alpha_{t}}\right)_{t \geq 0}$ is then called a continuously delayed filtration of $\mathbb{H}$.

One can easily check that $\mathbb{F}=\left(\mathcal{H}_{\alpha_{t}}\right)_{t \geq 0}$ is well-defined. See, for example, He, Wang, and Yan (1992, Chapter III, §5).

\footnotetext{
${ }^{1}$ This is also known as satisfying the usual hypotheses.

${ }^{2}$ That is, $\left(\alpha_{t}\right)_{t \geq 0}$ is an increasing, right continuous process, so that $\alpha_{0}=0$ and $\alpha_{t}$ is an $\mathbb{H}$-stopping time for every $t \geq 0$.
} 
Example 2. Given a filtration $\mathbb{H}=\left(\mathcal{H}_{t}\right)_{t \geq 0}$, a constant $\delta>0$, and let $\alpha_{t}=(t-\delta)^{+}$. Then, $\mathcal{F}_{t}=\mathcal{H}_{\alpha_{t}}$ defines a continuously delayed filtration of $\mathbb{H}$, so that

$$
\mathcal{F}_{t}=\left\{\begin{array}{r}
\mathcal{H}_{0}, t \leq \delta, \\
\mathcal{H}_{t-\delta}, t>\delta
\end{array}\right.
$$

This includes as a special case the example in Collin-Dufresne, Goldstein, and Helwege (2003, Appendix A1), where the filtration $\mathbb{H}$ is the natural filtration of a Brownian motion.

Next, we introduce

Definition 3 (Discretely delayed filtration). Given a filtration $\mathbb{H}=\left(\mathcal{H}_{t}\right)_{t \geq 0}$ that is right continuous and contains all the negligible sets. Suppose $\left(T_{n}^{k}\right)_{n \geq 0}(1 \leq k \leq K)$ are $K$ strictly increasing ${ }^{3}$ sequences of $\mathbb{H}$-stopping times. Suppose also $\left(\mathcal{G}_{i_{1} \cdots i_{K}}\right)_{i_{1}, \cdots, i_{K} \in \mathbb{N}}$ is a family of sub $\sigma$-field of $\mathcal{H}_{\infty}=$ $\vee_{t>0} \mathcal{H}_{t}$, so that

(i) $\mathcal{G}_{i_{1} \cdots i_{K}} \subset \mathcal{G}_{j_{1} \cdots j_{K}}$, if $i_{1} \leq j_{1}, \cdots, i_{K} \leq j_{K}$;

(ii) $\mathcal{G}_{i_{1} \cdots i_{K}} \subset \mathcal{H}_{T_{i_{1}}^{1} \vee \cdots \vee T_{i_{K}}^{K}}$, where $T_{i_{1}}^{1} \vee \cdots \vee T_{i_{K}}^{K}=\max \left\{T_{i_{1}}^{1}, \cdots, T_{i_{K}}^{K}\right\}$;

(iii) For any $k, T_{n}^{k}$ is $\mathcal{G}_{i_{1} \cdots i_{K}}$-measurable whenever $n \leq i_{k}$.

Define

$$
\mathcal{F}_{t}^{0}=\cup_{i_{1} \cdots i_{K}}\left(\mathcal{G}_{i_{1} \cdots i_{K}} \cap\left\{T_{i_{k}}^{k} \leq t<T_{i_{k}+1}^{k}: 1 \leq k \leq K\right\}\right)
$$

and

$$
\mathcal{F}_{t}=\cup_{i_{1} \cdots i_{K}}\left(\left(\mathcal{G}_{i_{1} \cdots i_{K}} \vee \sigma(\mathcal{N})\right) \cap\left\{T_{i_{k}}^{k} \leq t<T_{i_{k}+1}^{k}: 1 \leq k \leq K\right\}\right),
$$

where $\mathcal{N}$ is the collection of all negligible sets. Then $\mathbb{F}=\left(\mathcal{F}_{t}\right)_{t \geq 0}$ is a filtration that is right continuous and contains all the negligible sets, and is called a discretely delayed filtration of $\mathbb{H}$.

Its well-definedness can be seen from Proposition 16 in Appendix B.

Example 4. Suppose $X$ is a stochastic process and $\mathbb{H}=\left(\mathcal{H}_{t}\right)_{t \geq 0}$ is the augmented natural filtration of $X$. Let $\left(t_{k}\right)_{k \geq 0}$ be a strictly increasing sequence of non-negative numbers, and $\left(T_{n}\right)_{n \geq 0}$ a strictly increasing sequence of $\mathbb{H}$-stopping times. Then the minimal filtration generated by the two marked point processes $\left(t_{k}, X_{t_{k}}\right)_{k \geq 0}$ and $\left(T_{n}, X_{T_{n}}\right)_{n \geq 0}$ is a discretely delayed filtration.

For an increasing sequence $\left(t_{k}\right)_{k \geq 0}$ of deterministic times, Duffie and Lando (2001) considered the discrete observations $\left(Y_{t_{k}}\right)_{k \geq 0}$ of the process

$$
Y_{t}=z_{0}+m t+\sigma W_{t}+U_{t}
$$

where $W$ is a Brownian motion and $U$ is a Gaussian process independent of $W$.

Remark 5. Discretely delayed filtrations arise naturally from discrete observations, as from the above example. Mathematically speaking, the discretely delayed filtration is a generalization of the natural filtration of a marked point process, namely the jumping filtration in Jacod and Skorohod (1994). It is essentially the filtration generated by finitely many marked point processes.

\footnotetext{
${ }^{3} \mathrm{~A}$ sequence $\left(T_{n}\right)_{n \geq 0}$ of random variables is strictly increasing, if $T_{0}=0, T_{n} \uparrow \infty$ and $\forall n \geq 0, T_{n}<\infty \Longrightarrow T_{n}<$ $T_{n+1}$.
} 


\subsection{Distinction Between Continuously and Discretely Delayed Filtrations}

By definition, it is intuitively clear that the two types of delayed filtrations are distinct. To make this intuition precise, we first introduce the notion of a genuinely stochastic process.

Definition 6. Let $X$ be a Markov process whose state space $E$ is a metric space $(E, \rho) . X$ is genuinely stochastic, if $\forall x \in E$ and $t>0$,

(i) $X_{t}$ has continuous distribution under $P^{x}$;

(ii) $P^{x}\left(\rho\left(X_{t}, X_{0}\right)>\epsilon\right) \in(0,1)$ for any $\epsilon>0$;

(iii) $P^{x}\left(\rho\left(X_{t}, X_{0}\right)>\epsilon\right)$ is continuous in $\epsilon$.

Remark 7. By definition, we see that a stochastic process is genuinely stochastic if it genuinely leaves its starting position and does so in a continuous way. A sufficient condition for $X$ to be genuinely stochastic is that $X$ has a positive transition density function. Clearly, deterministic processes and Poisson processes are not genuinely stochastic. Meanwhile, a Brownian motion is genuinely stochastic. In fact, most processes of interest to financial applications are genuinely stochastic, including diffusion processes (with possible jumps) satisfying mild technical conditions. (For further details on existence of positive density functions for diffusion processes with jumps, interested readers may refer to [41], [42], [10], [9], [45], [39]).

It is no surprise that genuinely stochastic processes are the basis for many economics models: Asset prices are always fluctuating and not constant because information is continually happening in product markets. A company's asset value continually changes because it is being bombarded with new information from many different sources in many different locations regarding its inputs, outputs, production process, labor, competition, technology, economic setting, etc. In short, a very large number of weakly correlated and/or independent information sources are continually revealed, and change the asset price.

Now we show that for genuinely stochastic processes, the discretely delayed filtration and the continuously delayed filtration are distinct. Since genuinely stochastic processes include most "nondegenerate" diffusion processes, this distinction is important.

Theorem 8. Let $X$ be a genuinely stochastic strong Markov process, with $\mathbb{H}=\left(\mathcal{H}_{t}\right)_{t \geq 0}$ being its augmented natural filtration. If an $\mathbb{H}$-stopping time $T$ is not a constant, then the filtration $\mathbb{F}=\left(\mathcal{F}_{t}\right)_{t \geq 0}$, which is defined by $\mathcal{F}_{t}=\left(\mathcal{H}_{0} \cap\{t<T\}\right) \cup\left(\mathcal{H}_{T} \cap\{t \geq T\}\right)$, is a discretely delayed filtration of $\mathbb{H}$, but not a continuously delayed filtration of $\mathbb{H}$.

\section{Delayed Filtrations and Default Intensities}

Given the mathematical framework for incomplete information, this section shows how delayed filtrations generate default intensities for general Markov models: for models with or without jumps, and not necessarily diffusions.

Although the computation technique exploited here is the same as used in Duffie and Lando (2001) (for an alternative methodology to obtain closed-form formulas of intensity, see Guo and Zeng (2008)), there are a number of technical points worth noting. 
Computing the Intensity $\lambda_{t}$ from Aven's Theorem. Mathematically, the intensity process $\left(\lambda_{t}\right)_{t \geq 0}$ of a stopping time $\tau$ is associated with the compensator $A$ of $\tau$ with respect to a given filtration $\mathbb{G}$, relative to which $\tau$ is a stopping time. That is, an increasing, right-continuous, and adapted process $\left(A_{t}\right)_{t \geq 0}$ is called the $\mathbb{G}$-compensator of $\tau$, if $A_{0}=0,1_{\{\tau \leq t\}}-A_{t}$ is a $\mathbb{G}$-martingale and $A$ is $\mathbb{G}$-predictable. The intensity process $\left(\lambda_{t}\right)_{t \geq 0}$ of $\tau$ is then defined as the Radon-Nikodym derivative $\left(d A_{t} / d t\right)_{t \geq 0}$, provided that $A$ is a.s. absolutely continuous with respect to the Lebesgue measure. See Brémaud (1981).

Computing $\lambda_{t}$ via its associated compensator is in general not easy, except for some special cases. Here we adopt the the approach established by Aven (1985) through the Meyer's Laplacian approximation, as by Duffie and Lando (2001). The essence of this approach is to calculate the default intensity $\lambda_{t}$ by the intuitive definition

$$
\lambda_{t}=\lim _{h \downarrow 0} \frac{P\left(t+h \geq \tau>t \mid \mathcal{G}_{t}\right)}{h} .
$$

Here $\mathbb{G}=\left(\mathcal{G}_{t}\right)_{t}$ is an appropriate filtration for which $\tau$ is a stopping time. However, in general, this intuitive definition of $\lambda_{t}$ as the instantaneous likelihood of default given $\mathcal{G}_{t}$ is not necessarily the same as the Radon-Nikodym derivative of the compensator of $\tau$. Thus, the computation has to be verified using the Aven's Theorem. (See Appendix A for description of these mathematical tools, and Guo, Jarrow, and Menn (2006) for conditions under which these two notions are consistent).

Furthermore, this computational method for an intensity may fail, even for the following simple example.

Example 9. Let $W$ be a Brownian motion, $y>0$ a constant, and $\tau=\inf \left\{t>0: W_{t}>y\right\}$. Suppose $\left(h_{n}\right)_{n \geq 1}$ is a sequence of numbers decreasing to 0 , then under the natural filtration $\left(\mathcal{F}_{t}^{W}\right)_{t \geq 0}$ of $W$, we have

$$
\frac{1}{h_{n}} P\left(t+h_{n} \geq \tau>t \mid \mathcal{F}_{t}^{W}\right)=\frac{1}{h_{n}} 1_{\{\tau>t\}} P^{W_{t}}\left(\tau \leq h_{n}\right)=\frac{1_{\{\tau>t\}}}{h_{n}} \int_{0}^{h_{n}} \frac{\left|W_{t}-y\right|}{\sqrt{2 \pi t^{3}}} e^{-\frac{\left(W_{t}-y\right)^{2}}{2 t}} d t \stackrel{n \rightarrow \infty}{\longrightarrow} 0 .
$$

Since $\tau$ is predictable under the natural filtration $\left(\mathcal{F}_{t}^{W}\right)_{t \geq 0}$, the compensator of $\tau$ is $1_{\{\tau \leq t\}}$. Thus, 0 cannot be the intensity of $\tau$.

Clearly, in this example, Aven's conditions (i)-(iii) as in Theorem 15 (Appendix A) must be violated. Curiously, it is not trivial to see exactly why. (The proof is somewhat technical and delayed to Appendix D for interested readers).

Finally, note that for any non-negative random variable $\tau$ and a given filtration $\mathbb{F}=\left(\mathcal{F}_{t}\right)_{t \geq 0}, \tau$ is not necessarily an $\mathbb{F}$-stopping time. In this case, the intensity process $\left(\lambda_{t}\right)_{t \geq 0}$ of a stopping time $\tau$ is associated with the expanded filtration $\mathbb{G}=\left(\mathcal{G}_{t}\right)_{t \geq 0}$ of $\mathbb{F}=\left(\mathcal{F}_{t}\right)_{t \geq 0}$, where $\tau$ is a $\mathbb{G}$-stopping time. However, there is more than one way to expand $\mathbb{F}$. Issues regarding the consistency of different filtration expansions were studied by Guo and Zeng (2008). Throughout the paper, we adopt the simplest expansion approach known as the minimal filtration expansion. 


\subsection{The Continuously Delayed Filtration for a Continuous Strong Markov Pro- cess}

Let $X$ be a one-dimensional ${ }^{4}$, time homogeneous, continuous strong Markov process with $X_{0}=x$. Denote by $\mathbb{H}$ the augmented natural filtration of $X$. For a time change $\left(\alpha_{t}\right)_{t \geq 0}$ of $\mathbb{H}$, define $\mathbb{F}=\left(\mathcal{F}_{t}\right)_{t \geq 0}=\left(\mathcal{H}_{\alpha_{t}}\right)_{t \geq 0}$. The minimal expansion of $\mathbb{F}$ regarding $\tau$ is $\mathbb{G}=\left(\mathcal{G}_{t}\right)_{t \geq 0}$ with $\mathcal{G}_{t}=$ $\mathcal{F}_{t} \vee \sigma(\{\tau \leq s\}: s \leq t)$.

Theorem 10. Let $X$ be a one-dimensional, time homogeneous, continuous strong Markov process with $X_{0}=x$. Let $y<x$ and $\tau=\tau_{y}=\inf \left\{t>0: X_{t}<y\right\}$. Suppose $\tau$ has a density function $f(x, y, t) d t=P^{x}\left(\tau_{y} \in d t\right)$, and $f(x, y, t)$ is jointly continuous in $x, y$ and $t$ on $\{(x, y, t): x \neq y, t \geq$ $0\}$. If for a.s. $\omega, \alpha_{t}(\omega)<t$ for all $t>0$, then the $\mathbb{G}$-intensity of $\tau$ is

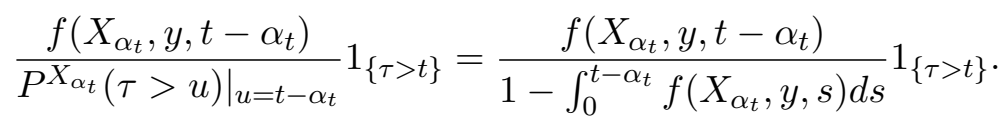

In particular, $\tau$ is a totally inaccessible $\mathbb{G}$-stopping time.

Example 11. [Collin-Dufresne et al. (2003)] Suppose $X$ is given by the Black-Scholes geometric Brownian motion model with parameters $\mu, \sigma$. For a positive constant $l>0$, the delayed filtration $\mathbb{F}=\left(\mathcal{F}_{t}\right)_{t \geq 0}$ with $\mathcal{F}_{t}=\sigma\left(W_{s}: s \leq t-l\right)$ is expanded to $\mathbb{G}=\left(\mathcal{G}_{t}\right)_{t \geq 0}$ with $\mathcal{G}_{t}=\mathcal{F}_{t} \vee \sigma(t \wedge \tau)$. Then on $\{\tau>t\}$, the default intensity of $\tau$ at time $t$ is $-\frac{\psi_{t}\left(\frac{\mu}{\sigma}-\frac{1}{2} \sigma, l, \frac{\log y-\log X_{t-l}}{\sigma}\right)}{\psi\left(\frac{\mu}{\sigma}-\frac{1}{2} \sigma, l, \frac{\log y-\log X_{t-l}}{\sigma}\right)}$, where $\psi(\theta, t, y)=$ $1-\int_{0}^{t} \frac{|y|}{\sqrt{2 \pi s^{3}}} e^{-\frac{(y-\theta s)^{2}}{2 s}} d s$ for $y<0$ and $\psi_{t}$ is the derivative of $\psi$ w.r.t. the time variable $t$.

More generally, if $X$ follows a one-dimensional stochastic differential equation $d X_{t}=\sigma\left(X_{t}\right) d W_{t}+$ $b\left(X_{t}\right) d t$ where $b$ and $\sigma$ satisfy some regularity conditions, then for $\tau_{y}$ (the first passage time of $X$ hitting the level $y$ starting from $x)$, there is a density function $f(x, y, t)$ such that $f(x, y, t)$ is jointly continuous (see Pauwels (1987) for details). Hence, the existence of the corresponding default intensity is implied, according to our result.

\subsection{The Discretely Delayed Filtration for a General Strong Markov Process}

Let $X$ be a time homogeneous strong Markov process. We denote by $\mathbb{H}=\left(\mathcal{H}_{t}\right)_{t \geq 0}$ the augmented natural filtration of $X$. We assume that $\mathbb{F}=\left(\mathcal{F}_{t}\right)_{t \geq 0}$ is a discretely delayed filtration of $\mathbb{H}$, as defined in Definition 3. Using the same notation as in Definition 3, we further require that $X_{T_{n}^{k}} \in \mathcal{G}_{i_{1} \cdots i_{K}}$ whenever $n \leq i_{k}$, and that each $T_{n}^{k}$ is finite. Finally, with a slight abuse of notation, we denote by $\mathbb{G}=\left(\mathcal{G}_{t}\right)_{t \geq 0}$ the minimal expansion of $\mathbb{F}$ with regard to $\tau$. A special case of this set up is when $\mathbb{F}$ is the natural filtration of $K$ marked point processes $\left(T_{n}^{k}, X_{T_{n}^{k}}\right)_{n \geq 0}(1 \leq k \leq K)$. Then $\mathbb{G}$ can be viewed as the natural filtration of $K+1$ marked point processes.

As in the case of a continuously delayed filtration, suppose $\tau$ is the first hitting time of a Borel subset $D$ of the state space. Denote by $\theta_{t}$ the shift operator (i.e. $X . \circ \theta_{t}=X_{t+}$.). We further assume the following property: each stopping time $T_{i}^{k}$, if not deterministic, satisfies

\footnotetext{
${ }^{4}$ The dimension requirement for $X$ is not essential, as $\tau$ can be replaced by the first hitting time of a Borel subset of the state space with proper metric.
} 
(i) $T_{i}^{k}>0$ a.s.;

(ii) for a.s. $\omega$ and for all $t \geq 0, T_{i+1}^{k}(\omega)>t \geq T_{i}^{k}(\omega) \Longrightarrow T_{i+1}^{k}(\omega)=t+T_{1}^{k} \circ \theta_{t}(\omega)$.

To calculate the intensity of $\tau$ under $\mathbb{G}$, we fix $i_{1}, \cdots, i_{K}$ and focus on the event $\left\{\tau>t, T_{i_{k}}^{k} \leq\right.$ $\left.t<T_{i_{k}+1}^{k}: 1 \leq k \leq K\right\}$.

Define $S=T_{i_{1}}^{1} \vee \cdots \vee T_{i_{K}}^{K}$ and $T=T_{i_{1}+1}^{1} \wedge \cdots \wedge T_{i_{K}+1}^{K}$. If none of $T_{i_{1}+1}^{1}, \cdots, T_{i_{K}+1}^{K}$ is deterministic, then on the event $\{S<T\}, T-S=V \circ \theta_{S}$ where $V=T_{1}^{1} \wedge \cdots \wedge T_{1}^{K} .{ }^{5}$ If at least one of $T_{i_{1}+1}^{1}, \cdots$, $T_{i_{K}+1}^{K}$ is deterministic, define $V$ as the minimum of those non-deterministic $T_{i_{k}+1}^{k}$ 's, and define $m$ as the minimum of those deterministic $T_{i_{k}+1}^{k}$ 's $(\inf \emptyset:=\infty)$. Then we have $T-S=V \circ \theta_{S} \wedge(m-S)$. Finally, we assume the function

$$
g(x, s, t):=\lim _{h \downarrow 0} \frac{1}{h} P^{x}(s+h \geq \tau>s, V>t)
$$

exists and is bounded and integrable in the $s$ variable.

Theorem 12. On the event $\left\{\tau>t, T_{i_{k}}^{k} \leq t<T_{i_{k}+1}^{k}: 1 \leq k \leq K\right\}$, the $\mathbb{G}$-intensity $\left(\lambda_{t}\right)_{t \geq 0}$ of $\tau$ is

$$
\left.\frac{g\left(X_{S}, r, r\right)}{P^{X_{S}}(\tau>r, V>r)}\right|_{r=t-S}
$$

\section{Examples and Applications}

In this section, we provide several more examples of incomplete information settings where intensity processes are obtained from structural models, under the minimal expansion $\mathbb{G}$ of a discretely delayed filtration $\mathbb{F}$, so that $\mathcal{G}_{t}=\mathcal{F}_{t} \vee \sigma(\tau \wedge t)$.

Another application is to compare risky debt pricing under complete and incomplete information.

\subsection{Examples}

A Regime Switching Process. In a regime-switching model, the firm's asset value process $X$ is assumed to follow a diffusion process given by

$$
d X_{t}=X_{t} \mu_{\epsilon(t)} d t+\sigma_{\epsilon(t)} X_{t} d W_{t}
$$

where $W$ is a standard one-dimensional Brownian motion, and $\epsilon$ is a finite-state continuous-time Markov chain, independent of $W$ and taking values $0,1, \cdots, S-1$ with a known generator $\left(q_{i j}\right)_{S \times S}$. Finally, the drift and volatility coefficients, $\mu(\cdot)$ and $\sigma(\cdot)$, are functions of $\epsilon$.

Now, let the discretely delayed filtration $\mathbb{F}=\left(\mathcal{F}_{t}\right)_{t \geq 0}$ be generated by the point process $\sum_{k=1}^{\infty} X_{t_{k}} 1_{\left\{t>t_{k}\right\}}$ and the marked point process $\left(\epsilon\left(T_{n}\right), X_{T_{n}}, T_{n}\right)_{n \geq 1}$. And let $\tau=\inf \left\{t>0: X_{t}<x\right\}$ for a fixed $x<X_{0}$. Then we have

- The stopping time $\tau$ is totally inaccessible under the filtration $\mathbb{G}$.

\footnotetext{
${ }^{5}$ To see this, fix $\omega \in\{S<T\}$ and note $T_{i_{k}}^{k}(\omega) \leq S(\omega)<T(\omega) \leq T_{i_{k}+1}^{k}(\omega), \forall k \in\{1,2, \cdots, K\}$. By assumption (ii), $T_{i_{k}+1}^{k}(\omega)=S(\omega)+T_{1}^{k} \circ \theta_{S}(\omega), \forall k \in\{1,2, \cdots, K\}$. Take minimum on both sides, we have $T(\omega)=S(\omega)+V\left(\theta_{S}(\omega)\right)$. So $T-S=V \circ \theta_{S}$.
} 
- Given any $t \in\left[t_{k}, t_{k+1}\right)$, if $\tau>t$ and $T_{n} \leq t<T_{n+1}$, the intensity process of $\tau$ is

$$
-\frac{\psi_{t}\left(\theta_{\epsilon(t)}, t-t_{k} \vee T_{n}, \frac{1}{\sigma_{\epsilon(t)}} \log \frac{x}{X_{t_{k} \vee T_{n}}}\right)}{\psi\left(\theta_{\epsilon(t)}, t-t_{k} \vee T_{n}, \frac{1}{\sigma_{\epsilon(t)}} \log \frac{x}{X_{t_{k} \vee T_{n}}}\right)},
$$

where

$$
\psi(\theta, t, y)=P\left(\inf _{0 \leq s \leq t} W_{s}^{(\theta)}>y\right)=1-\int_{0}^{t} \frac{|y|}{\sqrt{2 \pi s^{3}}} e^{-\frac{(y-\theta s)^{2}}{2 s}} d s \quad \text { for } y<0,
$$

with $W_{t}^{(\theta)}:=W_{t}+\theta t$, and $\psi_{t}$ being the derivative of $\psi$ w.r.t. the variable $t$.

A Jump Diffusion Process. Suppose that the firm's asset value process $X$ follows a jumpdiffusion model so that

$$
X_{t}=X_{0} e^{\left(\mu-\frac{1}{2} \sigma^{2}\right) t+\sigma W_{t}} \prod_{0<s \leq t, \Delta \epsilon(s) \neq 0} \xi_{\epsilon(s)},
$$

where $W$ is a standard one-dimensional Brownian motion, $\epsilon$ is a finite-state continuous-time Markov chain, independent of $W$ and taking values $0,1, \cdots, S-1$ with a known generator $\left(q_{i j}\right)_{S \times S}$, and $\Delta \epsilon(s):=\epsilon(s)-\epsilon(s-)$. We set $q_{i}=\sum_{j \neq i} q_{i j}$, and assign to each state $i$ of $\epsilon(0 \leq i \leq S-1)$ a positive random variable $\xi_{i}$ with distribution function $F_{i}$. Finally, we assume that $\left(\xi_{i}\right)_{i \geq 1}, \epsilon$ and $W$ are all independent.

In particular, if $\epsilon$ is a standard Poisson process, then $X$ satisfies the SDE

$$
\frac{d X_{t}}{X_{t-}}=\mu d t+\sigma d W_{t}+d\left(\sum_{n=1}^{\epsilon_{t}}\left(\xi_{\epsilon\left(T_{n}\right)}-1\right)\right)
$$

where $T_{n}$ is the $n$-th jump time of $\epsilon$.

Now, let us define the discretely delayed filtration $\mathbb{F}$ to be the filtration generated by the (marked) point processes $\sum_{n=0}^{\infty} X_{t_{k}} 1_{\left\{t_{k} \leq t\right\}}$ and $\left(\xi_{\epsilon\left(T_{n}\right)}, X_{T_{n}}, T_{n}\right)_{n \geq 1}$. And let $\tau=\inf \left\{t>0: X_{t}<x\right\}$ for a fixed $x<X_{0}$.

If $\tau>t, t_{k} \leq t<t_{k+1}$ and $T_{n} \leq t<T_{n+1}$, then the intensity process of $\tau$ is

$$
-\frac{\psi_{t}\left(\theta, t-t_{k} \vee T_{n}, \frac{1}{\sigma} \log \frac{x}{X_{t_{k} \vee T_{n}}}\right)}{\psi\left(\theta, t-t_{k} \vee T_{n}, \frac{1}{\sigma} \log \frac{x}{X_{t_{k} \vee T_{n}}}\right)}+\sum_{j \neq \epsilon(t)} q_{\epsilon(t) j} \frac{\int_{0}^{1} F_{j}(d z) \phi\left(\theta, t-t_{k} \vee T_{n}, \frac{1}{\sigma} \log \frac{x}{X_{t_{k} \vee T_{n}}}, \frac{1}{\sigma} \log \frac{x}{z X_{t_{k} \vee T_{n}}}\right)}{\psi\left(\theta, t-t_{k} \vee T_{n}, \frac{1}{\sigma} \log \frac{x}{X_{t_{k} \vee T_{n}}}\right)},
$$

where $\theta=\frac{\mu}{\sigma}-\frac{\sigma}{2}$,

$$
\psi(\theta, t, y)=P\left(\inf _{0 \leq s \leq t} W_{s}^{(\theta)}>y\right)=1-\int_{0}^{t} \frac{|y|}{\sqrt{2 \pi s^{3}}} e^{-\frac{(y-\theta s)^{2}}{2 s}} d s \text { for } y<0
$$

with $W_{t}^{(\theta)}:=W_{t}+\theta t$, and $\psi_{t}$ being the derivative of $\psi$ w.r.t. the variable $t$. For $y_{1} \leq y_{2}$,

$$
\begin{aligned}
& \phi\left(\theta, t, y_{1}, y_{2}\right) \\
= & P\left(\inf _{s \leq t} W_{s}^{(\theta)}>y_{1}, W_{t}^{(\theta)} \leq y_{2}\right) \\
= & \Phi\left(\frac{y_{2}-\theta t}{\sqrt{t}}\right)-\Phi\left(\frac{y_{1}-\theta t}{\sqrt{t}}\right)-e^{2 \theta y_{1}}\left[\Phi\left(\frac{y_{2}-2 y_{1}-\theta t}{\sqrt{t}}\right)-\Phi\left(\frac{-y_{1}-\theta t}{\sqrt{t}}\right)\right],
\end{aligned}
$$

with $\Phi(x)$ being the distribution of a standard normal random variable. 


\subsection{Risky Debt Pricing: Complete Information versus Delayed Information}

Now we explore risky debt pricing under complete and incomplete information. For simplicity, we focus on the relation between the values of a defaultable zero-coupon bond with a zero recovery rate under the two different filtration structures. Consider a zero-coupon bond issued by the firm paying $\$ 1$ at time $T$ if there is no default, and $\$ 0$ at time $T$ if the firm defaults prior to time $T$. For simplicity, we assume that the interest rate process is deterministic.

Now, let $X=\left(X_{t}\right)_{t \geq 0}$ be a general and multi-dimensional Markov process under a risk-neutral measure $Q$, with a general state space $E .^{6}$ Let $D$ be a subset of $E$ and $\tau=\inf \left\{t>0: X_{t} \in D\right\}$. Here, $X_{t}$ corresponds to the firm's asset value process, plus any additional processes needed for the interest rate and recovery rate process. Here again $\mathbb{H}=\left(\mathcal{H}_{t}\right)_{t \geq 0}$ the natural filtration of $X$.

Theorem 13. Let $\left\{t_{k}\right\}_{k \geq 0}$ be a strictly increasing sequence of non-negative numbers $\left(t_{0}=0\right)$, such that $t_{k} \uparrow \infty$. Under the filtration

$$
\mathcal{G}_{t}:=\sigma\left(X_{t_{1}}, \cdots, X_{t_{k}}\right) \vee \sigma(t \wedge \tau)
$$

for $t_{k} \leq t<t_{k+1}$, we have

$$
V^{\mathcal{B}}(t, T)=1_{\{\tau>t\}} \frac{V^{\mathcal{A}}\left(t_{k}, T\right)}{V^{\mathcal{A}}\left(t_{k}, t\right)} .
$$

Here $V^{\mathcal{A}}(t, T)=Q\left(\tau>T \mid \mathcal{H}_{t}\right)$ and $V^{\mathcal{B}}(t, T)=Q\left(\tau>T \mid \mathcal{G}_{t}\right)$.

Some simple remarks here. Clearly, $V^{\mathcal{A}}\left(t_{i}, T\right)$ differs from $V^{\mathcal{B}}\left(t_{i}, T\right)$ except at time $t_{i}(0 \leq i \leq k)$. Secondly, it is not necessary that $V^{\mathcal{A}}(t, T)>V^{\mathcal{B}}(t, T)$ or $V^{\mathcal{A}}(t, T)<V^{\mathcal{B}}(t, T)$. Indeed, when $t_{k}<t$, the latter price is based on the "dated" information $X_{t_{k}}$ as compared to the former. This dated and hence inaccurate information can generate either a higher or lower price. Finally, as $t \downarrow t_{k}, V^{\mathcal{B}}(t, T) \rightarrow V^{\mathcal{A}}(t, T)$, i.e., as the incomplete information gets updated, the corresponding price converges to that with complete information. It is worth noting that this relationship is independent of the risk-neutral measure under consideration.

\section{References}

[1] M. Ammann. Credit Risk Valuation. Springer, (2001).

[2] P. Artzner and F. Delbaen. Default risk insurance and incomplete market. Mathematical Finance, 5, 187-195, (1995).

[3] T. Aven. A theorem for determining the compensator of a counting process. Scandinavian Journal of Statistics, 12, 69-72, (1985).

[4] A. Bélanger, S. Shreve, and D. Wong. A general framework for pricing credit risk. Mathematical Finance, 14(3), 317-350, (2004).

[5] T. Bielecki and M. Rutkowski. Credit Risk: Modeling, Valuation, and Hedging. Springer, (2002).

\footnotetext{
${ }^{6}$ With regard to how the Markov property is invariant under a change of equivalent probability measure, one can consult Palmowski and Rolski (2002) for details.
} 
[6] F. Black and J. Cox. Valuing corporate securities: Some effects of bond indenture provisions. Journal of Finance, 31, 351-367, (1976).

[7] F. Black and M. Scholes. The pricing of options and corporate liabilities. Journal of Political Economy, 81, 637-654, (1973).

[8] C. Blanchet-Scalliet and M. Jeanblanc. Hazard rate for credit risk and hedging defaultable contingent claims. Finance and Stochastics, 8, 145-189, (2004).

[9] K. Bichteler, J-B Gravereaux, and J. Jacod. Malliavin Calulus for Processes with Jumps. Gordon and Breach, (1980).

[10] J-M. Bismut. Large Deviation and Malliavin Calculus. Birkhasuer, (1984).

[11] P. Brémaud. Point Processes and Queues: Martingale Dynamics. Springer-Verlag, New YorkBerlin, 1981.

[12] U. Çetin, R. Jarrow, P. Protter, and Y. Yildirim. Modeling credit risk with partial information. Annals of Applied Probability, 14, 1167-1178, (2004).

[13] S. Chava and R. Jarrow. Bankruptcy prediction with industry effects, Review of Finance, 8(4), 537-569, (2004).

[14] P. Collin-Dufresne, R. Goldstein, and J. Helwege. Is credit event risk priced? Modeling contagion via the updating of beliefs. Working paper, Carnegie Mellon University, (2003).

[15] N. Chen and S. G. Kou. Credit spreads, optimal capital structure, and implied volatility with endogenous default and jump risk. Preprint, (2005).

[16] Surveys confirm rapid market growth. Creditflux, issue 38, October 1, (2004).

[17] M. Davis. Hazard rates in the credit grades model. Unpublished manuscript, 2002.

[18] C. Dellacherie. Capacités et processus stochastiques. Springer-Verlag, Berlin-New York, 1972.

[19] G. R. Duffee. Estimating the price of default risk. The Review of Financial Studies, 12(1), 197-226, (1999).

[20] D. Duffie and D. Lando. Term structures and credit spreads with incomplete accounting information. Econometrica, 69, 633-664, (2001).

[21] D. Duffie, S. Schroder, and C. Skiadas. Recursive valuation of defaultable securities and the timing of resolution of uncertainty. Annals of Applied Probability, 6, 1075-1090, (1996).

[22] D. Duffie and K. Singleton. Modeling term structure of defaultable bonds. Review of Financial Studies, 12, 687-720, (1999).

[23] D. Duffie and K. Singleton, Credit Risk. Princeton University Press, (2003).

[24] D. Duffie and K. Wang. Multiperiod corporate failure prediction with stochastic covariates. Working paper, Stanford University, (2003). 
[25] R. Elliott, M. Jeanblanc, and M. Yor. On models of default risk. Mathematical Finance, 10, 179-195, (2000).

[26] K. Giesecke and L. Goldberg. Forecasting default in the face of uncertainty. Journal of Derivatives, 12(1), 14-25, (2004).

[27] X. Guo, R. Jarrow and C. Menn. A note on Lando's formula and condisitonal independence. Preprint, Cornell University, Available at http : //www.ieor.berkeley.edu/ xinguo/papers/II.C.5.pdf. (2006).

[28] X. Guo and Y. Zeng. Intensity process and compensator: A new filtration expansion approach and the Jeulin-Yor theorem. The Annals of Applied Probability, 18(1), 120-142, (2008).

[29] S. W. He, J. G. Wang and J. A. Yan. Semimartingale Theory and Stochastic Calculus. CRC Press, Boca Raton, (1992).

[30] J. Jacod and A. V. Skorohod. Jumping filtrations and martingales with finite variation. Séminaire de Probabilités XXVIII, 21-35, (1994).

[31] R. Jarrow, D. Lando, and S. Turnbull. A Markov model for the term structure of credit risk spread. Review of Financial Studies, 481-523, (1997).

[32] R. Jarrow and P. Protter. Structural versus reduced-form models: A new information based perspective. Journal of Investment Management, 2(2), 34-43, (2004).

[33] R. Jarrow and S. Turnbull. Credit Risk: Drawing the analogy. Risk Magazine, 5 (9), (1992).

[34] R. Jarrow and S. Turnbull. Pricing options of financial securities subject to default risk. Journal of Finance, 50, 53-86, (1995).

[35] M. Jeanblanc and M. Rutkowski. Default risk and hazard processes. Mathematical Finance Bachelier Conference 2000, 281-312. Springer, (2002).

[36] O. Kallenberg. Foundations of Modern Probability. Springer, 2nd edition, (2002).

[37] I. Karatzas and S. Shreve. Stochastic Calculus and Brownian Motion. Springer, 2nd edition, (1991).

[38] S. G. Kou and H. Wang. First passage times of a jump diffusion process. Advances in Applied Probability, Vol. 35, 504-531, (2003).

[39] Y. Ishikawa and H. Kunita. Malliavin calculus on the Wiener-Poisson space and its application to canonical SDE with jumps. Stochastic Process. Appl. 116(12), 1743-1769, (2006).

[40] S. Kusuoka. A remark on default risk models. Advances in Mathematical Economics, 1, 69-82, (1999).

[41] S. Kusuoka and D. Stroock. Application of the Malliavin Calculus I. Taniguchi Sympos SA Katata 271-306. (1982).

[42] S. Kusuoka and D. Stroock. Application of the Malliavin Calculus II. Journal of Faculty of Science, Univ. of Tokyo, Sec 1A, 32(1), 1-76, (1985). 
[43] D. Lando. Cox processes and credit-risky securities. Review of Derivative Research, 2, 99-120, (1998).

[44] D. Lando. Credit Risk Modeling: Theory and Applications. Princeton University Press, New Jersey, (2004).

[45] R. Léandre. Regularite de processus de sants degeneres. Ann. Inst. Henri Poincare, 21(2), (1985).

[46] F. Longstaff and E. Schwartz. A simple approach to valuing risky fixed and floating rate debt. Journal of Finance, 50, 789-819, (1985).

[47] P. A. Meyer. Probability and Potentials, Blasisdell Publishing Company, Massachusetts, (1966).

[48] D. Madan and H. Unal. Pricing the risk of default. Review of Derivative Research, 2, 121-160, (1995).

[49] R. Merton. On the pricing of corporate debt: The risk structure of interest rates. Journal of Finance, 29, 449-470, (1974).

[50] Z. Palmowski and T. Rolski. A technique for exponential change of measure for Markov processes. Bernoulli, 8(6), 767-785, (2002).

[51] E. J. Pauwels. Smooth first-passage densities for one-dimensional diffusions. Journal of Applied Probability, 24, 370-377. (1987).

[52] J. Pitman and M. Yor. Some divergent integrals of Brownian motion. Adv. in Appl. Probab. suppl., 109-116. (1986).

[53] P. Protter. Stochastic Integration and Differential Equations. Springer, New York, 2nd edition, (2004).

[54] D. Revuz and M. Yor. Continuous martingales and Brownian motion. erd edition. SpringerVerlag, Berlin, (1999).

[55] L. C. G. Rogers. Modeling credit risk. Preprint, University of Bath, (1999).

[56] L. C. G. Rogers and D. Williams. Diffusions, Markov Processes, and Martingales, Volume 2: Itô Calculus. Cambridge University Press, Cambridge, 2nd edition, (2000).

[57] P. Schönbucher. Credit Derivatives Pricing Models, John Wiley \& Sons, (2003).

[58] C. Zhou. A jump-diffusion approach to modeling credit risk and valuing defaultable securities. Technical report. Federal Research Board, Washington DC, (1997).

\section{Appendix A: Preliminaries}

Theorem 14 (Meyer's Laplacian Approximation). Let $Z$ be a right-continuous positive supermartingale of the class $(D)$ (i.e., the set $\left\{Z_{T}: T\right.$ is a stopping time satisfying $\left.T<\infty\right\}$ is uniformly integrable), with $\lim _{t \rightarrow \infty} E\left\{Z_{t}\right\}=0$. Let $Z=M-A$ be its Doob-Meyer decomposition. Define

$$
A_{t}^{h}=\int_{0}^{t} \frac{Z_{s}-E\left\{Z_{s+h} \mid \mathcal{F}_{s}\right\}}{h} d s .
$$


Then for any stopping time $T, A_{T}=\lim _{h \rightarrow 0} A_{T}^{h}$, in the sense of the weak topology $\sigma\left(L^{1}, L^{\infty}\right)$.

Proof. See Meyer (1966), page 119, T29.

The above theorem is used by T. Aven to prove the following theorem.

Theorem 15 (T. Aven, 1985). Let $\left(\Omega, \mathcal{F},\left(\mathcal{F}_{t}\right)_{t \geq 0}, P\right)$ be a filtered probability space that satisfies the usual hypotheses. Let $\left(N_{t}\right)_{t \geq 0}$ be a counting process. Assume that $E\left\{N_{t}\right\}<\infty$ for all $t$. Let $\left\{h_{n}\right\}_{n \geq 1}$ be a sequence which decreases to zero and let $\left(Y_{t}^{n}\right)_{t \geq 0}$ be a measurable version of the process $\left(E\left\{N_{t+h_{n}}-N_{t} \mid \mathcal{F}_{t}\right\} / h_{n}\right)_{t \geq 0}$ for each $n$. Assume that the following statements hold with $\left(\lambda_{t}\right)_{t \geq 0}$ and $\left(y_{t}\right)_{t \geq 0}$ being non-negative measurable processes:

(i) for each $t, \lim _{n} Y_{t}^{n}=\lambda_{t}$ a.s.;

(ii) for each $t$, there exists for almost all $\omega$ an $n_{0}=n_{0}(t, \omega)$ such that

$$
\left|Y_{s}^{n}(\omega)-\lambda_{s}(\omega)\right| \leq y_{s}(\omega), \quad s \leq t, n \geq n_{0}
$$

(iii) $\int_{0}^{t} y_{s} d s<\infty$, a.s. $0 \leq t<\infty$.

Then $N_{t}-\int_{0}^{t} \lambda_{s} d s$ is an $\mathcal{F}_{t}$-martingale, i.e., $\left(\int_{0}^{t} \lambda_{s} d s\right)_{t \geq 0}$ is the compensator of $\left(N_{t}\right)_{t \geq 0}$.

\section{Appendix B: Definition 3 is well defined}

Proposition 16. Adopting the notation in Definition 3, we have

1) $\mathbb{F}^{0}=\left(\mathcal{F}_{t}^{0}\right)_{t \geq 0}$ is a right-continuous sub-filtration of $\mathbb{H}$;

2) Each $T_{n}^{k}$ is a stopping time of $\mathbb{F}^{0}$;

3) $\mathbb{F}=\left(\mathcal{F}_{t}\right)_{t \geq 0}$ is the usual augmentation of $\mathbb{F}^{0}$.

Proof. 1) First, let us check that $\mathcal{F}_{t}^{0}$ is indeed a $\sigma$-field.

(i) $\Omega=\cup_{i_{1} \cdots i_{K}}\left(\Omega \cap\left\{T_{i_{k}}^{k} \leq t<T_{i_{k}+1}^{k}: 1 \leq k \leq K\right\}\right) \in \mathcal{F}_{t}^{0}$.

(ii) For any $A_{i_{1} \cdots i_{K}} \in \mathcal{G}_{i_{1} \cdots i_{K}}$,

$$
\begin{aligned}
& \left(\cup_{i_{1} \cdots i_{K}}\left(A_{i_{1} \cdots i_{K}} \cap\left\{T_{i_{k}}^{k} \leq t<T_{i_{k}+1}^{k}: 1 \leq k \leq K\right\}\right)\right)^{c} \\
= & \cap_{i_{1} \cdots i_{K}}\left(\left\{T_{i_{k}}^{k} \leq t<T_{i_{k}+1}^{k}: 1 \leq k \leq K\right\}^{c} \cup\left(A_{i_{1} \cdots i_{K}}^{c} \cap\left\{T_{i_{k}}^{k} \leq t<T_{i_{k}+1}^{k}: 1 \leq k \leq K\right\}\right)\right) \\
= & \cup_{i_{1} \cdots i_{K}}\left(A_{i_{1} \cdots i_{K}}^{c} \cap\left\{T_{i_{k}}^{k} \leq t<T_{i_{k}+1}^{k}: 1 \leq k \leq K\right\}\right) \\
\in & \mathcal{F}_{t}^{0} .
\end{aligned}
$$

(iii) If $A_{i_{1} \cdots i_{K}}^{(l)} \in \mathcal{G}_{i_{1} \cdots i_{K}}$, then

$$
\begin{aligned}
& \cup_{l} \cup_{i_{1} \cdots i_{K}}\left(A_{i_{1} \cdots i_{K}}^{(l)} \cap\left\{T_{i_{k}}^{k} \leq t<T_{i_{k}+1}^{k}: 1 \leq k \leq K\right\}\right) \\
= & \cup_{i_{1} \cdots i_{K}}\left(\left(\cup_{l} A_{i_{1} \cdots i_{K}}^{(l)}\right) \cap\left\{T_{i_{k}}^{k} \leq t<T_{i_{k}+1}^{k}: 1 \leq k \leq K\right\}\right) \\
\in & \mathcal{F}_{t}^{0} .
\end{aligned}
$$

Secondly, for any $A_{i_{1} \cdots i_{K}} \in \mathcal{G}_{i_{1} \cdots i_{K}}$,

$$
\begin{aligned}
& A_{i_{1} \cdots i_{K}} \cap\left\{T_{i_{k}}^{k} \leq t<T_{i_{k}+1}^{k}: 1 \leq k \leq K\right\} \\
\in & \mathcal{H}_{T_{i_{1}}^{1} \vee \cdots \vee T_{i_{K}}^{K}}^{K} \cap\left\{T_{i_{k}}^{k} \leq t<T_{i_{k}+1}^{k}: 1 \leq k \leq K\right\} \\
\subset \quad & \mathcal{H}_{t},
\end{aligned}
$$


implying $\mathcal{F}_{t}^{0} \subset \mathcal{H}_{t}$.

To see that $\left(\mathcal{F}_{t}^{0}\right)_{t \geq 0}$ is a filtration, for any $s<t$, let $A \in \mathcal{F}_{s}^{0}$ have the representation

$$
\cup_{i_{1} \cdots i_{K}}\left(A_{i_{1} \cdots i_{K}} \cap\left\{T_{i_{k}}^{k} \leq s<T_{i_{k}+1}^{k}: 1 \leq k \leq K\right\}\right)
$$

for some $A_{i_{1} \cdots i_{K}} \in \mathcal{G}_{i_{1} \cdots i_{K}}$. Then

$$
\begin{aligned}
& \left(\cup_{i_{1} \cdots i_{K}}\left(A_{i_{1} \cdots i_{K}} \cap\left\{T_{i_{k}}^{k} \leq s<T_{i_{k}+1}^{k}: 1 \leq k \leq K\right\}\right)\right) \cap\left\{T_{j_{k}}^{k} \leq t<T_{j_{k}+1}^{k}: 1 \leq k \leq K\right\} \\
= & \cup_{i_{1} \leq j_{1}, \cdots, i_{K} \leq j_{K}}\left(A_{i_{1} \cdots i_{K}} \cap\left\{T_{i_{k}}^{k} \leq s<T_{i_{k}+1}^{k}: 1 \leq k \leq K\right\} \cap\left\{T_{j_{k}}^{k} \leq t<T_{j_{k}+1}^{k}: 1 \leq k \leq K\right\}\right) \\
= & \cup_{i_{1} \leq j_{1}, \cdots, i_{K} \leq j_{K}}\left(A_{i_{1} \cdots i_{K}} \cap \cap \cap_{i_{k}<j_{k}}\left\{T_{i_{k}}^{k} \leq s<T_{i_{k}+1}^{k}\right\} \cap \cap_{i_{k}=j_{k}}\left\{T_{j_{k}}^{k} \leq s\right\}\right) \\
& \cap\left\{T_{j_{k}}^{k} \leq t<T_{j_{k}+1}^{k}: 1 \leq k \leq K\right\} \\
\in & \mathcal{G}_{j_{1} \cdots j_{K}} \cap\left\{T_{j_{k}}^{k} \leq t<T_{j_{k}+1}^{k}: 1 \leq k \leq K\right\} .
\end{aligned}
$$

Thus, $A \in \mathcal{F}_{t}^{0}$, implying $\mathcal{F}_{s}^{0} \subset \mathcal{F}_{t}^{0}$.

Finally, we show that $\mathbb{F}^{0}$ is right continuous. Let $h$ be $\cap_{n} \mathcal{F}_{t+\frac{1}{n}}^{0}$-measurable. Then for $\forall n \geq 1$,

$$
h=\sum_{i_{1} \cdots i_{K}} h_{i_{1} \cdots i_{K}}^{(n)} 1_{\left\{T_{i_{k}}^{k} \leq t+\frac{1}{n}<T_{i_{k}+1}^{k}: 1 \leq k \leq K\right\}}
$$

for some $h_{i_{1} \cdots i_{K}}^{(n)} \in \mathcal{G}_{i_{1} \cdots i_{K}}$. Define $h_{i_{1} \cdots i_{K}}=\lim \sup _{n} h_{i_{1} \cdots i_{K}}^{(n)} \in \mathcal{G}_{i_{1} \cdots i_{K}}$. Then for each $\omega \in\left\{T_{i_{k}}^{k} \leq t<\right.$ $\left.T_{i_{k}+1}^{k}: 1 \leq k \leq K\right\}$, there exists $n(\omega)$ such that $\forall m \geq n(\omega)$,

$$
\omega \in\left\{T_{i_{k}}^{k} \leq t+\frac{1}{m}<T_{i_{k}+1}^{k}: 1 \leq k \leq K\right\}
$$

This shows $h(\omega)=h_{i_{1} \cdots i_{K}}^{(n)}(\omega)=h_{i_{1} \cdots i_{K}}(\omega)$ for $\mathrm{n}$ large enough. That is,

$$
h=\sum_{i_{1} \cdots i_{K}} h_{i_{1} \cdots i_{K}} 1_{\left\{T_{i_{k}}^{k} \leq t<T_{i_{k}+1}^{k}: 1 \leq k \leq K\right\}} \in \mathcal{F}_{t}^{0},
$$

and hence $\mathcal{F}_{t}^{0}=\mathcal{F}_{t+}^{0}$.

2) To see that $T_{n}^{k}$ is an $\mathbb{F}^{0}$-stopping time, it suffices to note that $\left\{T_{n}^{k} \leq t\right\}=\cup_{i_{1} \cdots i_{k} \cdots i_{K}, i_{k} \geq n}\left\{T_{i_{l}}^{l} \leq\right.$ $\left.t<T_{i_{l}+1}^{l}: 1 \leq l \leq K\right\} \in \mathcal{F}_{t}^{0}$.

3 ) is obvious, since the operation of completion by negligible sets and the smallest extension to get right-continuity are commutable (see, for example, Kallenberg (2002), Lemma 7.8).

\section{Appendix C: Proof of Theorem 8}

To prove Theorem 8 , it is critical to establish the following lemma.

Lemma 17. Under the augmented natural filtration $\mathbb{H}=\left(\mathcal{H}_{t}\right)_{t \geq 0}$ of a genuinely stochastic strong Markov process $X$, if $\mathcal{H}_{S} \subset \mathcal{H}_{T}$ for stopping times $S$ and $T$, then $S \leq T P^{x}$-a.s. for all $x \in E$. In particular, stopping times $S$ and $T$ are equal $P^{x}$-a.s. for all $x \in E$ if and only if $\mathcal{H}_{S}=\mathcal{H}_{T}$. 
Proof of Lemma 17. By assumption, $S, X_{S} \in \mathcal{H}_{T}$. By the strong Markov property, for any $\epsilon>0$ and any $x \in E$, we have

$$
1_{\left\{S>T, \rho\left(X_{S}, X_{T}\right)>\epsilon\right\}}=1_{\{S>T\}} P^{x}\left(\rho\left(X_{S}, X_{T}\right)>\epsilon \mid \mathcal{H}_{T}\right)=\left.1_{\{S>T\}} P^{X_{T}}\left(\rho\left(X_{u}, X_{0}\right)>\epsilon\right)\right|_{u=S-T}, P^{x}-\text { a.s. }
$$

On the event $\{S>T\}$, the right hand side is between 0 and 1 , while the left hand side is either 0 or 1 . So we must have $P^{x}(S>T)=0$. Hence Lemma 17 .

Proof of Theorem 8. Obviously, $\mathbb{F}$ is a discretely delayed filtration of $\mathbb{H}$. Suppose $\mathbb{F}$ is also a continuously delayed filtration of $\mathbb{H}$, with some time change $\left(\alpha_{t}\right)_{t \geq 0}$. We show that $\alpha_{t}=T 1_{\{T \leq t\}}$ and obtain a contradiction.

Fix $t>0$ and $x \in E$. For any $A \in \mathcal{H}_{\alpha_{t}}=\mathcal{F}_{t}$, we have $A \cap\{t<T\} \in \mathcal{F}_{t} \cap\{t<T\}=\mathcal{H}_{0} \cap\{t<T\}$. By the Blumenthal's 0-1 law, if $A \cap\{t<T\}$ is not $P^{x}$-negligible, then it must be equal to $\{t<T\}$ $P^{x}$-a.s.

Now, we show that $\alpha_{t}=\alpha_{t} 1_{\{T \leq t\}} P^{x}$-a.s. Assume otherwise, then $P^{x}\left(t<T, \alpha_{t}>0\right)>0$. Therefore there exists $q \in(0, t)$ such that $P^{x}\left(t<T, \alpha_{t}>q\right)>0$. Define

$$
F(\epsilon)=P^{x}\left(t<T, \alpha_{t}>q, \rho\left(X_{q}, X_{0}\right) \leq \epsilon\right),
$$

then $F$ is continuous in $\epsilon, F(0)=0$ and $F(\infty)=P^{x}\left(t<T, \alpha_{t}>q\right)>0$. So there exists an $\epsilon_{0}$ such that $0<F\left(\epsilon_{0}\right)<P^{x}(t<T)$. On the other hand,

$$
\left\{\alpha_{t}>q, \rho\left(X_{q}, X_{0}\right) \leq \epsilon_{0}\right\} \in \mathcal{H}_{\alpha_{t}}=\mathcal{F}_{t},
$$

so $\left\{\alpha_{t}>q, \rho\left(X_{q}, X_{0}\right) \leq \epsilon_{0}, t<T\right\}=\{t<T\} P^{x}$-a.s. But,

$$
P^{x}\left(\alpha_{t}>q, \rho\left(X_{q}, X_{0}\right) \leq \epsilon_{0}, t<T\right)=F\left(\epsilon_{0}\right)<P^{x}(t<T) .
$$

This is a contradiction. We therefore conclude that $\alpha_{t}=\alpha_{t} 1_{\{T \leq t\}} P^{x}$-a.s.. By the right continuity of $\left(\alpha_{t}\right)_{t \geq 0}$, for $P^{x}$-a.s. $\omega, \alpha_{t}(\omega)=\alpha_{t}(\omega) 1_{\{T(\omega) \leq t\}}$, for any $t \geq 0$.

Secondly, it is easy to see $\mathcal{H}_{\alpha_{t}}=\mathcal{F}_{t} \subset \mathcal{H}_{T}$. By Lemma 17, $\alpha_{t} \leq T P^{x}$-a.s. $(\forall x \in E)$. Note $\{T \leq t\} \in \mathcal{F}_{t}=\mathcal{H}_{\alpha t}$, thus $\left(\alpha_{t}\right)_{\{T \leq t\}}:=\alpha_{t} 1_{\{T \leq t\}}+(+\infty) 1_{\{T>t\}}$ is an $\mathbb{H}$-stopping time. Define $T_{\{T \leq t\}}=T 1_{\{T \leq t\}}+(+\infty) 1_{\{T>t\}}$ and $\mathcal{H}_{T_{\{T \leq t\}}}=\left\{A \in \mathcal{H}_{\infty}: A \cap\left\{T_{\{T \leq t\}} \leq u\right\} \in \mathcal{H}_{u}, \forall u \geq 0\right\}$. Since

$$
\mathcal{H}_{T_{\{T \leq t\}}} \cap\{T>t\}=\mathcal{H}_{\infty} \cap\{T>t\}=\mathcal{H}_{\left(\alpha_{t}\right)_{\{T \leq t\}}} \cap\{T>t\}
$$

and

$$
\mathcal{H}_{T_{\{T \leq t\}}} \cap\{T \leq t\}=\mathcal{H}_{T} \cap\{T \leq t\}=\mathcal{F}_{t} \cap\{T \leq t\}=\mathcal{H}_{\alpha_{t}} \cap\{T \leq t\}=\mathcal{H}_{\left(\alpha_{t}\right)_{\{T \leq t\}}} \cap\{T \leq t\},
$$

we must have $\mathcal{H}_{T_{\{T \leq t\}}}=\mathcal{H}_{\left(\alpha_{t}\right)_{\{T \leq t\}}}$. By Lemma 17 and $\alpha_{t}=\alpha_{t} 1_{\{T \leq t\}}$, we conclude $\alpha_{t}=T 1_{\{T \leq t\}}$ $P^{x}$-a.s. for any $x \in E$. By the right-continuity of $\left(\alpha_{t}\right)_{t \geq 0}$, we conclude $\forall x \in E$, for $P^{x}$-a.s. $\omega$,

$$
\alpha_{t}(\omega)=T(\omega) 1_{\{T(\omega) \leq t\}}, \forall t \geq 0 .
$$

Now for any $u, t>0$ with $u<t,\left\{\alpha_{t} \leq u\right\} \in \mathcal{H}_{u}$. Since

$$
\left\{\alpha_{t} \leq u\right\}=\{T \leq t, T \leq u\} \cup\{T>t\}=\{T \leq u\} \cup\{T>t\}
$$

and $\{T \leq u\} \in \mathcal{H}_{u}$, we must have $\{T>t\} \in \mathcal{H}_{u}$. By letting $u \downarrow 0$, we have $\{T>t\} \in \cap_{u>0} \mathcal{H}_{u}=$ $\mathcal{H}_{0+}$. By the Blumenthal's 0-1 law, $T$ is a constant. Contradiction. 


\section{Appendix D: On Aven's condition for Example 11}

To see analytically how Aven's conditions are violated for Example 11, we introduce the following notion of mildly decreasing for $\left(h_{n}\right)_{n \geq 1}$.

Definition 18. Suppose $\left(h_{n}\right)_{n \geq 1}$ is a decreasing sequence of positive numbers. $\left(h_{n}\right)_{n \geq 1}$ is called mildly decreasing if there exists $a, b>0$ so that for $t$ sufficiently small, there is always some $h_{n}$ that lies between at and bt.

Using the notation introduced above, we shall see that, for the first passage time of a Brownian motion under its natural filtration, Aven's conditions do not hold when $\left(h_{n}\right)_{n \geq 1}$ is mildly decreasing.

First,

Lemma 19. Let $Y_{t}^{n}=1_{\{\tau>t\}} \frac{P^{W_{t}}\left(\tau \leq h_{n}\right)}{h_{n}}$, and assume $\left(h_{n}\right)_{n \geq 1}$ is mildly decreasing. Then Aven's condition (i)-(iii) hold if and only if

$$
\int_{0}^{\tau} \frac{d t}{\left(W_{t}-y\right)^{2}}<\infty, \text { a.s. }
$$

Proof. First, it is clear that $Y_{t}^{n} \rightarrow 0$ a.s. as $n \rightarrow \infty$. By change of variables, for $x \neq y$,

$$
P^{x}(\tau \leq h)=\int_{0}^{h} \frac{|x-y|}{\sqrt{2 \pi s^{3}}} e^{-\frac{(x-y)^{2}}{2 s}} d s=\frac{1}{\sqrt{2 \pi}} \int_{0}^{h /(x-y)^{2}} \frac{e^{-\frac{1}{2 u}}}{\sqrt{u^{3}}} d u=F\left(\frac{h}{(x-y)^{2}}\right),
$$

where $F(x)=\frac{1}{\sqrt{2 \pi}} \int_{0}^{x} \frac{e^{-\frac{1}{2 u}}}{\sqrt{u^{3}}} d u$. Note that $F(x)$ is strictly increasing with $F(0)=0$ and $F(\infty)<\infty$. Moreover,

$$
\lim _{x \rightarrow 0} \frac{F(x)}{x}=0 .
$$

So $F(x) / x$ is bounded away from 0 on any finite closed interval not containing 0 . Define $C=$ $\sup _{x>0} \frac{F(x)}{x}$, then we have

$$
Y_{t}^{n}=1_{\{\tau>t\}} \frac{1}{h_{n}} F\left(\frac{h_{n}}{\left(W_{t}-y\right)^{2}}\right) \leq 1_{\{\tau>t\}} \frac{C}{\left(W_{t}-y\right)^{2}}
$$

(Sufficiency) If $\int_{0}^{\tau} \frac{d t}{\left(W_{t}-y\right)^{2}}<\infty$, a.s., then we can simply set

$$
y_{t}:=1_{\{\tau>t\}} \frac{C}{\left(W_{t}-y\right)^{2}} .
$$

(Necessity) Assume that there is a set of positive probability so that on this set $\int_{0}^{\tau} \frac{d t}{\left(W_{t}-y\right)^{2}}=\infty$. Fix such an $\omega$, and choose $t_{0}$ such that $t_{0}>\tau(\omega)$. Then there exists $n_{0}$, so that for any $n \geq n_{0}$ and $t \leq t_{0}, Y_{t}^{n}(\omega) \leq y_{t}(\omega)$. Since $\left(h_{n}\right)_{n \geq 1}$ is mildly decreasing, there exist $a, b, \epsilon>0$, so that for any $x<\epsilon$, there is always some $h_{n}$ that lies between $a x$ and $b x$. In particular, we have $\left(t \leq t_{0}\right)$

$$
\begin{aligned}
& y_{t}(\omega) \\
\geq & \sup _{n \geq n_{0}} Y_{t}^{n}(\omega) \\
\geq & \sup _{n \geq n_{0}} 1_{\{\tau(\omega)>t\}} 1_{\left\{\left(W_{t}(\omega)-y\right)^{2}<\epsilon \wedge \frac{h_{n_{0}}(\omega)}{b}\right\}} \frac{1}{\left(W_{t}(\omega)-y\right)^{2}} \frac{F\left(\frac{h_{n}}{\left(W_{t}(\omega)-y\right)^{2}}\right)}{\frac{h_{n}}{\left(W_{t}(\omega)-y\right)^{2}}} \\
\geq & 1_{\{\tau(\omega)>t\}} 1_{\left\{\left(W_{t}(\omega)-y\right)^{2}<\epsilon \wedge \frac{h_{n_{0}}(\omega)}{b}\right\}} \frac{1}{\left(W_{t}(\omega)-y\right)^{2}} C^{\prime},
\end{aligned}
$$


where $C^{\prime}=\inf _{a \leq x \leq b} \frac{F(x)}{x}>0$. Hence

$$
\begin{aligned}
& \int_{0}^{t_{0}} y_{t}(\omega) d t \\
\geq & C^{\prime} \int_{0}^{\tau(\omega)} \frac{d t}{\left(W_{t}(\omega)-y\right)^{2}} d t-C^{\prime} \int_{0}^{\tau(\omega)} 1_{\left\{\left(W_{t}(\omega)-y\right)^{2} \geq \epsilon \wedge \frac{h_{n_{0}}(\omega)}{b}\right\}} \frac{1}{\left(W_{t}(\omega)-y\right)^{2}} d t \\
\geq & C^{\prime} \int_{0}^{\tau(\omega)} \frac{d t}{\left(W_{t}(\omega)-y\right)^{2}} d t-C^{\prime} \frac{\tau(\omega)}{\left(\epsilon \wedge \frac{h_{n_{0}}(\omega)}{b}\right)^{2}} \\
= & \infty .
\end{aligned}
$$

This shows that Aven's conditions will not hold on a set of positive probability.

Secondly,

Lemma 20.

$$
\int_{0}^{\tau} \frac{d t}{\left(W_{t}-y\right)^{2}}=\infty, \text { a.s. }
$$

Proof. According to [54] page 316, Corollary 4.6, it suffices to show the divergence of $\int_{0}^{\delta} \frac{1}{\rho_{t}^{2}} d t$, where $\delta$ is an arbitrarily small positive number and $\rho_{t}$ is a 3 -dimensional Bessel process starting from 0. It is known that $\int_{0}^{\delta} \frac{1}{\rho_{t}^{2}} d t=\infty$ a.s. from [52].

\section{Appendix E: Proof of Theorem 10}

Proof. By the strong Markov property and the general Bayes formula (see [25])

$$
\begin{aligned}
& \frac{1}{h} P\left(t<\tau \leq t+h \mid \mathcal{G}_{t}\right) \\
= & \frac{1}{h} \frac{P\left(t<\tau \leq t+h \mid \mathcal{F}_{t}\right)}{P\left(\tau>t \mid \mathcal{F}_{t}\right)} 1_{\{\tau>t\}} \\
= & \left.\frac{1}{h} \frac{P^{X_{\alpha_{t}}(u<\tau \leq u+h)}}{P^{X_{\alpha_{t}}(\tau>u)}} 1_{\{\tau>t\}}\right|_{u=t-\alpha_{t}} \\
\rightarrow & \lambda_{t}:=\left.\frac{f\left(X_{\alpha_{t}}, y, t-\alpha_{t}\right)}{P^{X_{\alpha_{t}}}(\tau>u)} 1_{\{\tau>t\}}\right|_{u=t-\alpha_{t}} \text { as } h \downarrow 0 .
\end{aligned}
$$

To show that $\lambda_{t}$ is indeed the intensity process of $\tau$, it remains to check Aven's conditions. Without loss of generality, we assume that $h \leq 1$. Then

$$
\begin{aligned}
& \left|\frac{1}{h} P\left(t<\tau \leq t+h \mid \mathcal{G}_{t}\right)-\lambda_{t}\right| \\
\leq & \left.\frac{1}{h} \frac{\int_{u}^{u+h}\left|f\left(X_{\alpha_{t}}, y, s\right)-f\left(X_{\alpha_{t}}, y, u\right)\right| d s}{P^{X_{\alpha_{t}}}(\tau>u)} 1_{\{\tau>t\}}\right|_{u=t-\alpha_{t}} \\
\leq & \frac{\sup _{t-\alpha_{t} \leq s \leq t-\alpha_{t}+1}\left|f\left(X_{\alpha_{t}}, y, s\right)-f\left(X_{\alpha_{t}}, y, t-\alpha_{t}\right)\right|_{\{\tau>t\}}}{\left.P^{X_{\alpha_{t}}}(\tau>u)\right|_{u=t-\alpha_{t}}} 1_{\left\{t_{t}\right.} \\
:= & y_{t} .
\end{aligned}
$$


Now it is easy to see for a.s. $\omega, \forall t_{0}>0, \int_{0}^{t_{0}} y_{t}(\omega) d t<\infty$. In fact, $y_{t}$ is bounded by a finite random variable on $[0, \tau)$ from the joint continuity of $f(x, y, t)$ on $\{(x, y, t): x \neq y, t \geq 0\}$.

\section{Appendix F: Proof of Theorem 12}

Proof. Note $\mathcal{G}_{t} \cap\left\{\tau>t, T_{i_{k}}^{k} \leq t<T_{i_{k}+1}^{k}: 1 \leq k \leq K\right\}=\mathcal{G}_{i_{1} \cdots i_{K}} \cap\left\{\tau>t, T_{i_{k}}^{k} \leq t<T_{i_{k}+1}^{k}: 1 \leq k \leq\right.$ $K\}$. So by Bayes' formula, we have

$$
\begin{aligned}
& P\left(t<\tau \leq t+h \mid \mathcal{G}_{t}\right) 1_{\left\{T_{i_{k}}^{k} \leq t<T_{i_{k}+1}^{k}: 1 \leq k \leq K\right\}} \\
= & 1_{\left\{\tau>t, T_{i_{k}}^{k} \leq t<T_{i_{k}+1}^{k}: 1 \leq k \leq K\right\}} \frac{P\left(t<\tau \leq t+h, T_{i_{k}}^{k} \leq t<T_{i_{k}+1}^{k}: 1 \leq k \leq K \mid \mathcal{G}_{i_{1} \cdots i_{K}}\right)}{P\left(\tau>t, T_{i_{k}}^{k} \leq t<T_{i_{k}+1}^{k}: 1 \leq k \leq K \mid \mathcal{G}_{i_{1} \cdots i_{K}}\right)} .
\end{aligned}
$$

On the event $\left\{\tau>t, T_{i_{k}}^{k} \leq t<T_{i_{k}+1}^{k}: 1 \leq k \leq K\right\}$, we have (note $t-S<m-S$ is always true)

$$
\begin{aligned}
& \frac{1}{h} P\left(t<\tau \leq t+h \mid \mathcal{G}_{t}\right) \\
= & \frac{1}{h} \frac{P\left(t<\tau \leq t+h, S \leq t<T \mid \mathcal{G}_{i_{1} \cdots i_{K}}\right)}{P\left(\tau>t, S \leq t<T \mid \mathcal{G}_{i_{1} \cdots i_{K}}\right)} \\
= & \frac{1}{h} \frac{P\left(t<\tau \leq t+h, t-S<\left(V \circ \theta_{S}\right) \wedge(m-S) \mid \mathcal{G}_{i_{1} \cdots i_{K}}\right)}{P\left(\tau>t, t-S<\left(V \circ \theta_{S}\right) \wedge(m-S) \mid \mathcal{G}_{i_{1} \cdots i_{K}}\right)} \\
= & \frac{1}{h} \frac{P\left(t-S<\tau \circ \theta_{S} \leq t+h-S, t-S<V \circ \theta_{S} \mid \mathcal{G}_{i_{1} \cdots i_{K}}\right)}{P\left(\tau \circ \theta_{S}>t-S, t-S<V \circ \theta_{S} \mid \mathcal{G}_{i_{1} \cdots i_{K}}\right)} \\
= & \left.\frac{1}{h} \frac{P^{X_{S}}(r+h \geq \tau>r, V>r)}{P^{X_{S}}(\tau>r, V>r)}\right|_{r=t-S} \\
\rightarrow & \frac{g\left(X_{S}, r, r\right)}{\left.P^{X_{S}(\tau>r, V>r)}\right|_{r=t-S} .}
\end{aligned}
$$

The third equality holds because of the following: since $\tau$ is the first hitting time of a Borel subset $D$ of the state space, on the event $\{S<\tau\}, \tau(\omega)=.\inf \left\{t>0: \omega_{t} \in D\right\}=\inf \left\{t>S(\omega):. \omega_{t} \in\right.$ $D\}=S(\omega)+.\inf \left\{t>0: \omega_{S(\omega .)+t} \in D\right\}=S(\omega)+.\inf \left\{t>0: \theta_{S}(\omega .)_{t} \in D\right\}=S(\omega)+.\tau \circ \theta_{S}(\omega$.$) .$ Thus on the event $\{S<\tau\}, \tau-S=\tau \circ \theta_{S}$.

Now, similarly to the proof of Theorem 10, one can verify the Aven's condition by finding a dominating process and prove the theorem.

\section{Appendix G: Proof of Theorem 13}

Proof. Suppose $t \in\left[t_{k}, t_{k+1}\right)$, then

$$
\begin{aligned}
& V^{\mathcal{B}}(t, T)=1_{\{\tau>t\}} \frac{Q\left(\tau>T \mid X_{t_{1}}, \cdots, X_{t_{k}}\right)}{Q\left(\tau>t \mid X_{t_{1}}, \cdots, X_{t_{k}}\right)} \\
= & 1_{\{\tau>t\}} \frac{Q\left(1_{\left\{\tau>t_{k}\right\}} Q\left(X_{s} \notin D, \forall s \in\left[t_{k}, T\right] \mid \mathcal{H}_{t_{k}}\right) \mid X_{t_{1}}, \cdots, X_{t_{k}}\right)}{Q\left(1_{\left\{\tau>t_{k}\right\}} Q\left(X_{s} \notin D, \forall s \in\left[t_{k}, t\right] \mid \mathcal{H}_{t_{k}}\right) \mid X_{t_{1}}, \cdots, X_{t_{k}}\right)} \\
= & 1_{\{\tau>t\}} \frac{Q\left(X_{s} \notin D, \forall s \in\left[t_{k}, T\right] \mid X_{t_{k}}\right)}{Q\left(X_{s} \notin D, \forall s \in\left[t_{k}, t\right] \mid X_{t_{k}}\right)}=1_{\{\tau>t\}} \frac{V^{\mathcal{A}}\left(t_{k}, T\right)}{V^{\mathcal{A}}\left(t_{k}, t\right)} .
\end{aligned}
$$

